\title{
Agonistic postures in the rat: Reliability of human observations
}

\author{
D. A. POWELL, J. R. HOLLEY, and S. L. BUCHANAN \\ Neuroscience Laboratory, VA Hospital, Columbia, South Carolina 29201 \\ and
}

University of South Carolina, Columbia, South Carolina 29201

\begin{abstract}
Trained observers independently observed and recorded several preselected agonistic and other social postures in one experiment studying spontaneous aggression and three experiments involving shock-elicited aggression. Phi coefficients were calculated for each posture, based on agreement vs. nonagreement between observers. These reliability coefficients were significant for all postures in all four experiments. However, the strength of the relationship was consistently greater for different postures. Agreement for individual postures was also somewhat lower than has been reported for a fight vs. no-fight dichotomy, but was considered to be useful for many experimental purposes.
\end{abstract}

There has been a veritable explosion of the literature on aggression in recent years. This research includes investigations which range over the entire spectrum of biochemical, physiological, and situational variables. Similarly, many innovative techniques for assessing agonistic behaviors have been developed. Several of these techniques have focused upon objective measurements which remove the experimenter from the situation (e.g., Azrin, Hutchinson, \& Hake, 1966). However, a large number of studies have attempted to record, via human observers, agonistic postures executed by various species of animals (e.g., Conner \& Levine, 1969). Almost all of the latter research has focused upon the manipulation of independent variables, thus giving short shrift to obvious measurement problems associated with human observers. Although the usefulness of using such observers cannot be overemphasized, the reliability of these observations should be adequately assessed.

The present research was oriented toward evaluating human interrater reliability using two kinds of aggression models with rodents. These models included (a) the "free-cage model," in which two animals are simply placed in an enclosure devoid of other stimulation, and (b) the "shock aggression" model, in which electric shock is administered to elicit fighting.

\section{METHOD}

\section{Subjects}

Both Sprague-Dawley albino (Flow Laboratories) and LongEvans hooded (Blue Spruce Farms) rats were employed as subjects. All animals were maintained on ad-lib food and water in

This research was supported by VA Institutional Research Funds, Project No. 7155-01. Request reprints from D. A. Powell. Neuroscience Laboratory, Veterans Administration Hospital, Columbia. South Carolina 29201. suspended rodent cages. The rodent room was maintained on a 7:00 a.m.-7:00 p.m. light-dark cycle. All animals were run during the daylight portion of this cycle.

\section{Apparatus}

One experimental chamber, hereafter referred to as the "small" chamber, was a standard Scientific Prototype rodent enclosure. This chamber was housed inside a deactivated refrigerator shell with a one-way mirror for visual observation of the animal. When subcutaneous shock was employed, the experimental enclosure was a $58.4 \times 45.7 \mathrm{~cm}$ plywood box equipped with a mercury commutator arrangement for shock delivery and a Plexiglas front for visual observation. This chamber, hereafter referred to as the "large" chamber, was also equipped with a shock grid, and is described more fully in Duncan and Powell (1973) and Holley and Powell (1976). A $B R S$ shock generator and scrambler was used to present shock to both chambers. The chambers also contained 12-in. speakers, over which $75 \mathrm{~dB}$ white noise was presented, for sound attenuation. $B R S$ digibits and timing circuits provided programming for all experiments.

\section{Procedure}

Free-cage aggression. Twenty-four male and 24 female LongEvans rats were paired (within sex) in the smaller enclosure for either $10 \mathrm{~min}$ (eight pairs of each sex) or $16 \mathrm{~min}$ (four pairs of each sex). Each animal of a pair was observed on alternate minutes by two observers who independently recorded the frequency of occurrence of each of the agonistic postures described below. Although a frequency count of each behavioral category was made during each $1-\mathrm{min}$ trial, only the occurrence or nonoccurrence of the behavior during a trial was subjected to statistical analysis. The latter procedure was employed since many postures had a zero frequency for several animals (see Table 1), and product moment correlation coefficients were thus precluded. In addition, reliability during the shock-aggression experiments was assessed by computing phi coefficients. It was thus thought that a similar analysis for the free-cage experiment would facilitate comparisons between the two procedures.

Shock-elicited aggression. Three different experiments were performed in which shock was used to elicit agonistic posturing. In the first experiment, 12 male Sprague-Dawley and 12 male Long-Evans rats were paired (within strains) in the larger chamber and presented subcutaneous shock through previously im- 
Table 1

Phi Coefficients Relating Frequency of Agreement and Nonagreement to Observations of Agonistic Postures Made by Two Independent Observers

\begin{tabular}{|c|c|c|}
\hline Response & Phi Coefficient & $\begin{array}{r}\text { Proportion of } \\
\text { trials on which } \\
\text { response } \\
\text { occurred }\end{array}$ \\
\hline Crouching/Locomotion & .82 & .96 \\
\hline Upright, nonthreat & .86 & .92 \\
\hline Sniffing & .73 & .44 \\
\hline Autogroom & .93 & .24 \\
\hline Allogroom & .76 & .38 \\
\hline Crawling over/under & .75 & .11 \\
\hline Mutual upright (Threat) & .68 & .11 \\
\hline Striking & .66 & .01 \\
\hline Biting & .75 & .02 \\
\hline Dominance & 1.0 & .03 \\
\hline Submission & 1.0 & .03 \\
\hline Attack & 1.0 & .03 \\
\hline Lateral Block & * & .01 \\
\hline
\end{tabular}

*Expected frequencies too low to calculate.

Note-Proportion of 1-min trials on which each posture occurred is also shown.

planted electrodes (see Duncan \& Powell, 1973). Shocks consisted of .5 -sec trains presented at a 3 -sec intertrial interval. Onehundred shocks were presented during each session. Three different shock intensities were employed for two sessions each. These included $0,1,2$, and $3 \mathrm{~mA}$. These intensities were presented in a random order over consecutive daily sessions. Again. two trained observers recorded each agonistic posture which occurred during the 3 -sec interval following the shock.

In the second experiment. 10 pairs of male and 10 pairs of female Sprague-Dawley animals were placed in the smaller chamber and $3-\mathrm{mA} .5-\mathrm{sec}$ shock trains administered through the grid to the subject's feet with an intertrial interval of $3 \mathrm{sec}$. Each session consisted of 100 shock trials. After each shock, the behavior of each member of the pair was independently recorded by two observers as described below.

In the final experiment Long-Evans rats were presented with either (a) footshock. (b) subcutaneous shock, or (c) no shock, in the larger chamber. All shocks were 2-mA .5-sec duration trains presented with a 3-sec intershock interval. The three shock conditions were presented once each to six pairs of animals according to a Latin square design. The Latin square was replicated once with six additional pairs of subjects. Each session was recorded on video tape and observations later made by two trained sbservers who independently recorded the frequency of the postures described below. The observers had varying degrees of training. However, all had been exposed to at least six 5-min sessions in which shock was used to elicit aggression, and during which the various postures elicited were pointed out by an observer with extensive experience with agonistic behaviors in rodents (DAP).

It should be noted that all postures were not recorded in every experiment. The original descriptions of most of these behaviors can be found in the monograph by Grant and Mackintosh (1963), and in Barnett (1963). More recent descriptions of these, as well as other social behaviors, appear in Miczek and Barry (1974), Powell, Silverman. Francis and Schneiderman (1970), Spigel and Fraser (1974). etc. It should be noted that many social postures, not usually categorized as agonistic, were also recorded in these experiments. The behaviors recorded are listed below:

Nonfighting behaviors: (1) Nonsocial behaviors-"crouching/ locomotion"-both animals had all four paws on the grid floor, with no indication of social interaction. In the free-cage situation. this response also included locomotor behaviors typical of the investigatory behavior of the rat. (2) "Sniffing"-sniffing of the ano-genital region or nose of the opponent. (3) Each animal was in a crouching to semi-upright posture with its long body axis at $90 \mathrm{deg}$ to $180 \mathrm{deg}$ and in contact by its nose. Did not include sniffing of the head region. (4) "Crawling" over/ under-One pair member assumed a crouching posture while the other walked over or under it. (5) "Allogroom"-Vigorous sniffing and grooming of the head, nose, and shoulder, and at times flank regions of the other pair member. (6) "Self-grooming"Licking and sniffing of the paws, ano-genital region, etc.; preening behavior. (7) "Upright, nonthreat"-One pair member assumed a standing upright posture with forepaws off the floor, but was not oriented toward its opponent. (8) "Jumping"-One pair member jumped into the air with all the paws leaving the chamber floor, an escape response typically seen in rodents in response to aversive stimulaton.

Fighting postures: (1) Mutually upright ("threat")-The animal assumed an upright posture and was oriented toward the opponent, which was usually also upright, but occasionally might assume a half-crouching defensive posture with one forelimb raised and the body arched. (2) "Striking"-Mutual upright postures with boxing blows of the forepaws delivered to the head, neck, chest of the opponent. (3) "Biting"-Tooth contact to the head, neck, or flank areas of the opponent. (4) "Attack"A leaping charge terminating in upright-threat, striking, or more usually, a dominant posture with the opponent assuming a submissive posture. (5) "Sideways attack" or "lateral block"-One pair member assumed a 90-deg angle with respect to the opponent with the long body axis curved toward the opponent; one of the forepaws may be raised or may be used for attack (6) "Dominance-submission"-One member of the pair assumed a supine posture with its opponent in an upright and/or striking or biting posture standing above. This posture has been referred to as the "full attack" posture.

\section{RESULTS AND DISCUSSION}

Reliability coefficients (Phi coefficients) were computed for each category of behavior. These coefficients were computed from 2 by 2 contingency tables, composed of the frequency with which the posture involved was recorded or not recorded by each observer. Thus each posture, on a given trial, was (a) recorded by both $\mathrm{Ob}$ server 1 and Observer 2 (in which case agreement occurred), (b) was recorded by Observer 1 but not by Observer 2 (disagreement occurred), (c) was recorded by Observer 2 but not by Observer 1 (disagreement also occurred), or (d) was not recorded by either Observer 1 or Observer 2 (in which case agreement again occurred). These coefficients are shown in Table 1 for the experiment in which free-cage aggression was studied. The proportion of 1-min trials on which a response occurred is also shown in Table 1. Dominance-submission and attack had the highest coefficients, followed by crouching, upright, and grooming. Other coefficients were intermediate. However, all coefficients were significant $(p<.001)$.

The reliability coefficients for the three experiments involving shock elicited aggression are shown in Tables 2 4. As can be seen, these coefficients do not differ greatly from those shown in Table 1. Again, crouching, upright, and striking were more reliably identified than other postures, but as was found in the experiment on free-cage aggression, all coefficients were significant $(p<.001)$. The behavior in Experiment 4 , on shock 
Table 2

Phi Coefficients Relating Frequency of Agreement and Nonagreement to Observations of Agonistic Postures Made by $T$ wo Independent Observers

\begin{tabular}{lcc} 
Response & & $\begin{array}{r}\text { Proportion of } \\
\text { trials on which } \\
\text { response }\end{array}$ \\
Crouching/Locomotion & .80 & .44 \\
Upright (nonthreat) & .85 & .35 \\
Mutual upright (Threat) & .54 & .06 \\
Striking & .78 & .11 \\
Biting & .61 & .03 \\
Dominance & .75 & .06 \\
Submission & .72 & .05 \\
Jumping & .65 & .04 \\
Preening & .57 & .01 \\
Nosing & .48 & .06 \\
Crawl over/under & .59 & .02 \\
\hline
\end{tabular}

Note-Proportion of trials on which each posture occurred is also shown. The behavior was elicited by subdermal shock presented through previously implanted flank electrodes.

Table 3

Phi Coefficients Relating Frequency of Agreement and Nonagreement to Observations of Agonistic Postures Made by Two Independent Observers

\begin{tabular}{lcc} 
Response & Phi Coefficient & $\begin{array}{r}\text { on which response } \\
\text { occurred }\end{array}$ \\
Crouching/Locomotion & .91 & .19 \\
Upright, nonthreat & .65 & .28 \\
Jumping & .69 & .07 \\
Mutual upright (Threat) & .51 & .15 \\
Striking & .85 & .35 \\
Biting & .76 & .04 \\
\hline
\end{tabular}

Note-Proportion of trials on which each posture occurred is also shown. The behavior was elicited by footshock.

Table 4

Phi Coefficients Relating Frequency of Agreement and Nonagreement to Observations of Agonistic Postures Made by Two Independent Observers

\begin{tabular}{lcc}
\hline & \multicolumn{2}{c}{$\begin{array}{r}\text { Proportion of trials on } \\
\text { which response } \\
\text { occurred }\end{array}$} \\
Response & Phi Coefficient & .02 \\
Crosing & .43 & .36 \\
Upright (nonthreat) & .74 & .24 \\
Jumping & .59 & .05 \\
Crawl over/under & .76 & .01 \\
Dominance-submission & .48 & .02 \\
Mutual upright (Threat) & .68 & .07 \\
Striking & .44 & .17 \\
Biting & .76 & .03 \\
Full attack posture & .50 & .02 \\
\hline
\end{tabular}

Note-Proportion of trials on which each posture occurred is also shown. The behavior was elicited by subdermal shock and the observations made from video tape recordings. elicited aggression, was recorded on video tape and later observed and categorized in an effort to improve the reliability of observations in Experiments 1, 2, and 3 . However, the reliability coefficients computed on these data were not noticeably higher, and for some postures (e.g., crouching and upright) were in fact somewhat lower than those of Experiments 2 and 3.

Although these reliability coefficients were all highly significant, they are not especially high compared to some other kinds of behaviors. They are, in fact, much lower for some postures than has been previously reported for aggression in rodents (e.g., Miczek \& Barry, 1974). The consistently lower reliability of certain postures (e.g., threat and nosing) suggest that these responses should perhaps be omitted in future studies, or combined with other categories. Even though the coefficients of the present experiment are somewhat lower than desired, and are definitely lower than one obtains with a fight vs. no-fight dichotomy (Duncan \& Powell, 1975; Ulrich \& Azrin, 1962), their statistical significance suggests that these behaviors may be useful for many experimental purposes. For example, where experimental manipulations would be expected to differentially affect attack vs. defense, or perhaps other specific social postures (e.g., grooming), they could be quite useful, although not as reliable as a fight vs. no-fight dichotomy. Although all the observers who participated in the present investigation were trained in the same laboratory, the varying amounts of training to which they were previously exposed suggests that the results can be generalized to other laboratories. In all, seven different observers were used in the present study, three of whom had only limited exposure to the task (see procedure above). The similarity of the coefficients over all four experiments in which different observers were used further attests to the generality of these results.

Although the major purpose of the present investigation was to assess observer reliability, note might also be made of the proportion of trials during which different postures were recorded. These proportions, shown in Experiments 1, 2, and 3, sum to greater than zero since the different postures were not mutually exclusive, viz, it was possible to record more than a single response per trial. Fighting postures were not observed as frequently in the free-cage situation as in the footshock or subdermal shock situations. This is not especially notable since laboratory rats have been specifically inbred for nonaggression. That such postures occur at all, in the absence of aversive stimulation, is perhaps more notable.

Of greater importance is the finding that a wide range of different agonistic postures were elicited by both footshock and subdermal shock. The pattern of these behaviors should also be noted. The higher frequencies of defensive postures in all shock situations (e.g., upright threat and striking) are compatible with the characterization of these behaviors as primarily defensive and thus basically different from other animal models of 
aggression (e.g., those that feature attack behavior or possibly a combination of attack and defense). However, the occurrence of virtually all naturally occurring agonistic postures in response to shock suggest that aversive stimuli elicit agonistic response systems identical to those which operate in more naturalistic situations.

\section{REFERENCES}

Azrin. N. H.. Hetchinson, R. R.. \& Hake. D. F. Extinction induced aggression. Journal of Experimental Analysis of Behavior. 1906. 9. 191-204.

BARNETT. S. A. The rat: $A$ study in behavior. Chicago: Aldine, 1903.

Conner. R. L.. \& Levine, S. S. Garattini \& E. B. Sig (Eds.). Hormonal influences on aggressive behavior. New York: Wiley. 1969. Pp. 150-103.

Dencan. S. D.. d Powell. D. A. An inexpensive apparatus for presenting asersive stimulation to rodents in a social situation. Psychological Reports. 1973. 32. 851-854.

Duncan. S. D.. \& Powell. D. A. Agonistic postures elicited by subdermal shock in Sprague-Dawley and Long-Evans rats. Psychological Reports. 1975, 36. 327-337.

Grant, E. C., \& Mackintosh. J. H. A comparison of the social postures of some common laboratory rodents. Behavior. 1963. 21. $246-259$.

Holley, J. R.. \& Powell. D. A. Mercury commutator arrangement for simultaneously stimulating or recording from iwo small animals in a social situation. Physiology and Behotior. 19\%6, 15, $71,-43$.

MiczeK. K. A.. \& BARRY. H. delta-9-Tetrahydrocannabinol and aggressive behavior in rats. Behavioral Biology. 1974. 11. 261.207.

Powell, D. A.. Silverman. T., Francis, J.. \& Schneiderman. $\mathrm{N}$. The effects of sex and prior experience with fighting on shockinduced aggression. Communications in Bchavioral Biology: $14^{-0} 0.5$. 51-56.

SPIGEL. I. M..\& Fraser. D. "Dominance" in the laboratory rat: - The emergence of grooming. Zeitschrift fur Psychologie, 1974. 34. 59-69.

Ulrich. R. E.. \& Azrin. N. H. Reflexive fighting in response to aversive stimulation. Journal of Experimental Analysis of Behavior. 1962. 5. $511-520$.

(Received for publication January 16. 1976.) 